DESY-04-037

29th October 2018

\title{
Study of the pion trajectory in the photoproduction of leading neutrons at HERA
}

\author{
ZEUS Collaboration
}

\begin{abstract}
Energetic neutrons produced in ep collisions at HERA have been studied with the ZEUS detector in the photoproduction regime at a mean photonproton center-of-mass energy of $220 \mathrm{GeV}$. The neutrons carry a large fraction $0.64<x_{L}<0.925$ of the incoming proton energy, and the fourmomentum-transfer squared at the proton-neutron vertex is small, $|t|<$ $0.425 \mathrm{GeV}^{2}$. The $x_{L}$ distribution of the neutrons is measured in bins of t. The $\left(1-x_{L}\right)$ distributions in the $t$ bins studied satisfy a power law $d N / d x_{L} \propto\left(1-x_{L}\right)^{a(t)}$, with the powers $a(t)$ following a linear function of $t: \quad a(t)=0.88 \pm 0.09$ (stat. $)_{-0.39}^{+0.34}$ (syst.) $-(2.81 \pm 0.42 \text { (stat. })_{-0.62}^{+1.13}$ (syst.) $\left.\mathrm{GeV}^{-2}\right) t$. This result is consistent with the expectations of pion-exchange models, in which the incoming proton fluctuates to a neutron-pion state, and the electron interacts with the pion.
\end{abstract}




\section{The ZEUS Collaboration}

S. Chekanov, M. Derrick, D. Krakauer, J.H. Loizides ${ }^{1}$, S. Magill, S. Miglioranzi ${ }^{1}$, B. Musgrave, J. Repond, R. Yoshida

Argonne National Laboratory, Argonne, Illinois 60439-4815, USA ${ }^{n}$

M.C.K. Mattingly

Andrews University, Berrien Springs, Michigan 49104-0380, USA

P. Antonioli, G. Bari, M. Basile, L. Bellagamba, D. Boscherini, A. Bruni, G. Bruni,

G. Cara Romeo, L. Cifarelli, F. Cindolo, A. Contin, M. Corradi, S. De Pasquale, P. Giusti,

G. Iacobucci, A. Margotti, A. Montanari, R. Nania, F. Palmonari, A. Pesci, G. Sartorelli,

A. Zichichi

University and INFN Bologna, Bologna, Italy ${ }^{e}$

G. Aghuzumtsyan, D. Bartsch, I. Brock, S. Goers, H. Hartmann, E. Hilger, P. Irrgang, H.-P. Jakob, O. Kind, U. Meyer, E. Paul'², J. Rautenberg, R. Renner, A. Stifutkin, J. Tandler ${ }^{3}$, K.C. Voss, M. Wang

Physikalisches Institut der Universität Bonn, Bonn, Germany ${ }^{b}$

D.S. Bailey ${ }^{4}$, N.H. Brook, J.E. Cole, G.P. Heath, T. Namsoo, S. Robins, M. Wing

H.H. Wills Physics Laboratory, University of Bristol, Bristol, United Kingdom ${ }^{m}$

M. Capua, A. Mastroberardino, M. Schioppa, G. Susinno

Calabria University, Physics Department and INFN, Cosenza, Italy ${ }^{e}$

J.Y. Kim, I.T. Lim, K.J. Ma, M.Y. Pac ${ }^{5}$

Chonnam National University, Kwangju, South Korea ${ }^{g}$

A. Caldwell ${ }^{6}$, M. Helbich, X. Liu, B. Mellado, Y. Ning, S. Paganis, Z. Ren, W.B. Schmidke, F. Sciulli

Nevis Laboratories, Columbia University, Irvington on Hudson, New York 10027 o

J. Chwastowski, A. Eskreys, J. Figiel, A. Galas, K. Olkiewicz, P. Stopa, L. Zawiejski

Institute of Nuclear Physics, Cracow, Poland ${ }^{i}$

L. Adamczyk, T. Bołd, I. Grabowska-Bołd ${ }^{7}$, D. Kisielewska, A.M. Kowal, M. Kowal, J. Łukasik, M. Przybycień, L. Suszycki, D. Szuba, J. Szuba ${ }^{8}$

Faculty of Physics and Nuclear Techniques, AGH-University of Science and Technology, Cracow, Poland ${ }^{p}$

A. Kotański ${ }^{9}$, W. Słomiński

Department of Physics, Jagellonian University, Cracow, Poland 
V. Adler, U. Behrens, I. Bloch, K. Borras, V. Chiochia, D. Dannheim ${ }^{10}$, G. Drews, J. Fourletova, U. Fricke, A. Geiser, P. Göttlicher ${ }^{11}$, O. Gutsche, T. Haas, W. Hain, S. Hillert ${ }^{12}$, C. Horn, B. Kahle, U. Kötz, H. Kowalski, G. Kramberger, H. Labes, D. Lelas, H. Lim, B. Löhr, R. Mankel, I.-A. Melzer-Pellmann, C.N. Nguyen, D. Notz, A.E. NuncioQuiroz, A. Polini, A. Raval, L. Rurua, U. Schneekloth, U. Stösslein, G. Wolf, C. Youngman, W. Zeuner

Deutsches Elektronen-Synchrotron DESY, Hamburg, Germany

S. Schlenstedt

DESY Zeuthen, Zeuthen, Germany

G. Barbagli, E. Gallo, C. Genta, P. G. Pelfer

University and INFN, Florence, Italy ${ }^{e}$

A. Bamberger, A. Benen, F. Karstens, D. Dobur, N.N. Vlasov

Fakultät für Physik der Universität Freiburg i.Br., Freiburg i.Br., Germany ${ }^{b}$

M. Bell, P.J. Bussey, A.T. Doyle, J. Ferrando, J. Hamilton, S. Hanlon, D.H. Saxon, I.O. Skillicorn

Department of Physics and Astronomy, University of Glasgow, Glasgow, United Kingdom $^{m}$

I. Gialas

Department of Engineering in Management and Finance, Univ. of Aegean, Greece

T. Carli, T. Gosau, U. Holm, N. Krumnack, E. Lohrmann, M. Milite, H. Salehi, P. Schleper, T. Schörner-Sadenius, S. Stonjek ${ }^{12}$, K. Wichmann, K. Wick, A. Ziegler, Ar. Ziegler Hamburg University, Institute of Exp. Physics, Hamburg, Germany ${ }^{b}$

C. Collins-Tooth, C. Foudas, R. Gonçalo ${ }^{13}$, K.R. Long, A.D. Tapper

Imperial College London, High Energy Nuclear Physics Group, London, United King$d_{\text {om }}^{m}$

P. Cloth, D. Filges

Forschungszentrum Jülich, Institut für Kernphysik, Jülich, Germany

M. Kataoka ${ }^{14}$, K. Nagano, K. Tokushuku ${ }^{15}$, S. Yamada, Y. Yamazaki

Institute of Particle and Nuclear Studies, KEK, Tsukuba, Japan ${ }^{f}$

A.N. Barakbaev, E.G. Boos, N.S. Pokrovskiy, B.O. Zhautykov

Institute of Physics and Technology of Ministry of Education and Science of Kazakhstan, Almaty, Kazakhstan

D. Son

Kyungpook National University, Center for High Energy Physics, Daegu, South Korea ${ }^{g}$ 
K. Piotrzkowski

Institut de Physique Nucléaire, Université Catholique de Louvain, Louvain-la-Neuve, Belgium

F. Barreiro, C. Glasman ${ }^{16}$, O. González, L. Labarga, J. del Peso, E. Tassi, J. Terrón, M. Zambrana

Departamento de Física Teórica, Universidad Autónoma de Madrid, Madrid, Spain ${ }^{l}$

M. Barbi, F. Corriveau, S. Gliga, J. Lainesse, S. Padhi, D.G. Stairs, R. Walsh

Department of Physics, McGill University, Montréal, Québec, Canada H3A 2T8 ${ }^{a}$

T. Tsurugai

Meiji Gakuin University, Faculty of General Education, Yokohama, Japan ${ }^{f}$

A. Antonov, P. Danilov, B.A. Dolgoshein, D. Gladkov, V. Sosnovtsev, S. Suchkov Moscow Engineering Physics Institute, Moscow, Russia ${ }^{j}$

R.K. Dementiev, P.F. Ermolov, I.I. Katkov, L.A. Khein, I.A. Korzhavina, V.A. Kuzmin, B.B. Levchenko, O.Yu. Lukina, A.S. Proskuryakov, L.M. Shcheglova, S.A. Zotkin

Moscow State University, Institute of Nuclear Physics, Moscow, Russia ${ }^{k}$

N. Coppola, S. Grijpink, E. Koffeman, P. Kooijman, E. Maddox, A. Pellegrino, S. Schagen, H. Tiecke, M. Vázquez, L. Wiggers, E. de Wolf NIKHEF and University of Amsterdam, Amsterdam, Netherlands ${ }^{h}$

N. Brümmer, B. Bylsma, L.S. Durkin, T.Y. Ling

Physics Department, Ohio State University, Columbus, Ohio $43210^{n}$

A.M. Cooper-Sarkar, A. Cottrell, R.C.E. Devenish, B. Foster, G. Grzelak, C. Gwenlan ${ }^{17}$, T. Kohno, S. Patel, P.B. Straub, R. Walczak

Department of Physics, University of Oxford, Oxford United Kingdom ${ }^{m}$

A. Bertolin, R. Brugnera, R. Carlin, F. Dal Corso, S. Dusini, A. Garfagnini, S. Limentani, A. Longhin, A. Parenti, M. Posocco, L. Stanco, M. Turcato Dipartimento di Fisica dell' Università and INFN, Padova, Italy ${ }^{e}$

E.A. Heaphy, F. Metlica, B.Y. Oh, J.J. Whitmore ${ }^{18}$

Department of Physics, Pennsylvania State University, University Park, Pennsylvania $16802^{\circ}$

Y. Iga

Polytechnic University, Sagamihara, Japan ${ }^{f}$

G. D'Agostini, G. Marini, A. Nigro

Dipartimento di Fisica, Università 'La Sapienza' and INFN, Rome, Italy ${ }^{e}$ 
C. Cormack ${ }^{19}$, J.C. Hart, N.A. McCubbin

Rutherford Appleton Laboratory, Chilton, Didcot, Oxon, United Kingdom ${ }^{m}$

C. Heusch

University of California, Santa Cruz, California 95064, USA ${ }^{n}$

I.H. Park

Department of Physics, Ewha Womans University, Seoul, Korea

N. Pavel

Fachbereich Physik der Universität-Gesamthochschule Siegen, Germany

H. Abramowicz, A. Gabareen, S. Kananov, A. Kreisel, A. Levy

Raymond and Beverly Sackler Faculty of Exact Sciences, School of Physics, Tel-Aviv

University, Tel-Aviv, Israel ${ }^{d}$

M. Kuze

Department of Physics, Tokyo Institute of Technology, Tokyo, Japan ${ }^{f}$

T. Fusayasu, S. Kagawa, T. Tawara, T. Yamashita

Department of Physics, University of Tokyo, Tokyo, Japan ${ }^{f}$

R. Hamatsu, T. Hirose ${ }^{2}$, M. Inuzuka, H. Kaji, S. Kitamura ${ }^{20}$, K. Matsuzawa

Tokyo Metropolitan University, Department of Physics, Tokyo, Japan ${ }^{f}$

M. Costa, M.I. Ferrero, V. Monaco, R. Sacchi, A. Solano

Università di Torino and INFN, Torino, Italy ${ }^{e}$

M. Arneodo, M. Ruspa

Università del Piemonte Orientale, Novara, and INFN, Torino, Italy ${ }^{e}$

T. Koop, J.F. Martin, A. Mirea

Department of Physics, University of Toronto, Toronto, Ontario, Canada M5S 1A' a

J.M. Butterworth ${ }^{21}$, R. Hall-Wilton, T.W. Jones, M.S. Lightwood, M.R. Sutton ${ }^{4}$, C. TargettAdams

Physics and Astronomy Department, University College London, London, United Kingdom $^{m}$

J. Ciborowski22, R. Ciesielski ${ }^{23}$, P. Lużniak $^{24}$, R.J. Nowak, J.M. Pawlak, J. Sztuk ${ }^{25}$,

T. Tymieniecka, A. Ukleja, J. Ukleja ${ }^{26}$, A.F. Żarnecki

Warsaw University, Institute of Experimental Physics, Warsaw, Poland ${ }^{q}$

M. Adamus, P. Plucinski

Institute for Nuclear Studies, Warsaw, Poland ${ }^{q}$

Y. Eisenberg, D. Hochman, U. Karshon M. Riveline

Department of Particle Physics, Weizmann Institute, Rehovot, Israel ${ }^{c}$ 
A. Everett, L.K. Gladilin ${ }^{27}$, D. Kçira, S. Lammers, L. Li, D.D. Reeder, M. Rosin, P. Ryan, A.A. Savin, W.H. Smith

Department of Physics, University of Wisconsin, Madison, Wisconsin 53706, USA ${ }^{n}$

A. Deshpande, S. Dhawan

Department of Physics, Yale University, New Haven, Connecticut 06520-8121, USA ${ }^{n}$

S. Bhadra, C.D. Catterall, S. Fourletov, G. Hartner, S. Menary, M. Soares, J. Standage

Department of Physics, York University, Ontario, Canada M3J 1P3 ${ }^{a}$ 
${ }^{1}$ also affiliated with University College London, London, UK

2 retired

3 self-employed

4 PPARC Advanced fellow

${ }^{5}$ now at Dongshin University, Naju, South Korea

${ }^{6}$ now at Max-Planck-Institut für Physik, München, Germany

${ }^{7}$ partly supported by Polish Ministry of Scientific Research and Information Technology, grant no. 2P03B 12225

${ }^{8}$ partly supported by Polish Ministry of Scientific Research and Information Technology, grant no.2P03B 12625

${ }^{9}$ supported by the Polish State Committee for Scientific Research, grant no. 2 P03B 09322

${ }^{10}$ now at Columbia University, N.Y., USA

11 now at DESY group FEB

12 now at University of Oxford, Oxford, UK

13 now at Royal Holoway University of London, London, UK

14 also at Nara Women's University, Nara, Japan

15 also at University of Tokyo, Tokyo, Japan

16 Ramón y Cajal Fellow

17 PPARC Postdoctoral Research Fellow

18 on leave of absence at The National Science Foundation, Arlington, VA, USA

19 now at University of London, Queen Mary College, London, UK

20 present address: Tokyo Metropolitan University of Health Sciences, Tokyo 116-8551, Japan

21 also at University of Hamburg, Alexander von Humboldt Fellow

22 also at Eódź University, Poland

23 supported by the Polish State Committee for Scientific Research, grant no. 2P03B 07222

24 Łódź University, Poland

25 Łódź University, Poland, supported by the KBN grant 2P03B12925

26 supported by the KBN grant 2P03B12725

27 on leave from MSU, partly supported by the Weizmann Institute via the U.S.-Israel BSF 
a supported by the Natural Sciences and Engineering Research Council of Canada (NSERC)

$b$ supported by the German Federal Ministry for Education and Research (BMBF), under contract numbers HZ1GUA 2, HZ1GUB 0, HZ1PDA 5, HZ1VFA 5

c supported by the MINERVA Gesellschaft für Forschung GmbH, the Israel Science Foundation, the U.S.-Israel Binational Science Foundation and the Benozyio Center for High Energy Physics

$d$ supported by the German-Israeli Foundation and the Israel Science Foundation

$e$ supported by the Italian National Institute for Nuclear Physics (INFN)

$f$ supported by the Japanese Ministry of Education, Culture, Sports, Science and Technology (MEXT) and its grants for Scientific Research

$g$ supported by the Korean Ministry of Education and Korea Science and Engineering Foundation

$h$ supported by the Netherlands Foundation for Research on Matter (FOM)

$i$ supported by the Polish State Committee for Scientific Research, grant no. 620/E-77/SPB/DESY/P-03/DZ 117/2003-2005

$j$ partially supported by the German Federal Ministry for Education and Research (BMBF)

$k$ supported by RF President grant N 1685.2003.2 for the leading scientific schools and by the Russian Ministry of Industry, Science and Technology through its grant for Scientific Research on High Energy Physics

$l$ supported by the Spanish Ministry of Education and Science through funds provided by CICYT

$m$ supported by the Particle Physics and Astronomy Research Council, UK

$n$ supported by the US Department of Energy

$o$ supported by the US National Science Foundation

$p$ supported by the Polish Ministry of Scientific Research and Information Technology, grant no. 112/E-356/SPUB/DESY/P-03/DZ 116/2003-2005

$q$ supported by the Polish State Committee for Scientific Research, grant no. 115/E-343/SPUB-M/DESY/P-03/DZ 121/2001-2002, 2 P03B 07022 


\section{Introduction}

Several studies of leading neutron production in ep interactions at HERA have been reported previously [1-5]. Many features of the data are described by pion exchange models, in which the incoming proton fluctuates into a neutron-pion state and the pion interacts with the incoming electron or positron. The kinematic variables $t$, the square of the four-momentum transfer at the proton-neutron vertex, and $x_{L}$, the energy fraction of the proton carried by the neutron, are convenient variables for studying energy-angle correlations. In this study of semi-inclusive photoproduction, $\gamma p \rightarrow n X$, where the photon is quasi-real, the energy distribution of leading neutrons is measured as a function of $t$, which is determined using a new position detector to measure the angle of the neutron. The $\left(1-x_{L}\right)$ distribution is presented as a function of $t$ for large $x_{L}\left(0.64<x_{L}<0.925\right)$ and small $|t|\left(|t|<0.425 \mathrm{GeV}^{2}\right)$. The results are interpreted in the context of pion exchange, in order to provide a test of the consistency of this picture of leading neutron production.

\section{Experimental set-up and kinematics}

The data used for this measurement were collected in the year 2000 at the ep collider HERA with the ZEUS detector, during a short run period in which a special trigger was implemented. The data set corresponds to an integrated luminosity of $9 \mathrm{pb}^{-1}$. During this period HERA collided $27.5 \mathrm{GeV}$ positrons with $920 \mathrm{GeV}$ protons at a center-of-mass energy of $318 \mathrm{GeV}$.

Charged particles are tracked in the central tracking detector [6], which operates in a magnetic field of $1.43 \mathrm{~T}$ provided by a thin superconducting solenoid and covers the polarangle ${ }^{1}$ region $15^{\circ}<\theta<164^{\circ}$. Outside the solenoid is a hermetic, high-resolution uraniumscintillator calorimeter (CAL) [7] used to measure the energies of the final-state particles.

Bremsstrahlung $e p \rightarrow e \gamma p$ and the photoproduction of hadrons $e p \rightarrow e X$ are tagged using the luminosity (LUMI) detectors [8]. The bremsstrahlung photons are measured with a lead-scintillator calorimeter located $107 \mathrm{~m}$ from the interaction point in the positron-beam direction. A similar calorimeter at $35 \mathrm{~m}$ from the interaction point is used to measure positrons scattered at very small angles in an energy range 5-20 GeV, with an energy resolution of $0.19 \sqrt{E}(E$ in $\mathrm{GeV})$.

\footnotetext{
${ }^{1}$ The ZEUS coordinate system is a right-handed Cartesian system, with the $Z$ axis pointing in the proton beam direction, referred to as the "forward direction", and the $X$ axis pointing left towards the center of HERA. The coordinate origin is at the nominal interaction point.
} 
ZEUS has two detectors for measuring forward particles, a leading proton spectrometer (LPS) [9], consisting of six stations of silicon strip detectors, and a forward neutron calorimeter (FNC) [10-12] downstream from the LPS, $105 \mathrm{~m}$ from the interaction point. The FNC is a lead-scintillator sampling calorimeter, the front section of which is divided into 14 towers, as illustrated in Fig. 1. The hadronic energy resolution, as measured in a pion test beam at $120 \mathrm{GeV}$, is $0.65 \sqrt{E}(E$ in $\mathrm{GeV})$. Three planes of veto counters, located 70, 78, and $199 \mathrm{~cm}$ in front of the calorimeter, are used to detect charged particles produced by neutrons interacting in inactive material in front of the FNC.

Magnet apertures limit the FNC geometric acceptance to neutrons inside the area outlined in Fig. 1. This limit corresponds to neutron production angles less than 0.8 mrad; i.e., to transverse momenta $p_{T}=E_{n} \theta_{n} \leq 0.74 x_{L} \mathrm{GeV}$. The neutron production angle, $\theta_{n}$, is measured with respect to the proton beam direction at the interaction point. The mean value of $p_{T}$ of the detected neutrons is $0.22 \mathrm{GeV}$. The overall acceptance of the FNC is about $25 \%$ for neutrons with energy $x_{L}>0.6$ and $\theta_{n}<0.8 \mathrm{mrad}$.

The forward neutron tracker (FNT), designed to measure the position of neutron showers, was installed in the FNC in 1998. It consists of two hodoscope planes of $1.2 \mathrm{~cm}$-wide scintillator strips, with 17 strips measuring the $X$ position and 15 strips measuring the $Y$ position. Figure 1 shows the position of the FNT hodoscope in the FNC in the plane transverse to the beam as well as the zero-degree point, the projection of the proton beam direction at the interaction point onto the FNT. The FNT is situated approximately one interaction length inside the FNC. This position is deep enough that a large fraction of the neutrons begin to shower in front of the FNT, but not so deep as to compromise the position resolution. The position resolution of neutron showers in the FNT was measured to be $0.23 \mathrm{~cm}$ by placing an adjustable collimator in front of the FNC during special test and calibration runs.

The kinematics of inclusive photoproduction $e p \rightarrow e \gamma p \rightarrow e X$ at HERA is specified by $W$, the photon-proton center-of-mass energy. This is related to the positron-proton centerof-mass energy, $\sqrt{s}$, by $W^{2}=y s$. The inelasticity, $y$, of the scattered positron is defined by $y=\left(E-E^{\prime}\right) / E$, where $E\left(E^{\prime}\right)$ is the energy of the incoming (scattered) positron.

To describe the neutron-tagged process $e p \rightarrow e \gamma p \rightarrow e n X$, the variables $x_{L}$ and $p_{T}$ are also used. These are related to $t$ by

$$
t \approx-\frac{p_{T}^{2}}{x_{L}}-\frac{\left(1-x_{L}\right)}{x_{L}}\left(m_{n}^{2}-x_{L} m_{p}^{2}\right)
$$

where $m_{p}$ is the mass of the proton and $m_{n}$ is the mass of the neutron. 


\section{Event selection}

The data sample was collected using a trigger that required at least $5 \mathrm{GeV}$ in the LUMI electron calorimeter in coincidence with at least $0.5 \mathrm{GeV}$ in the rear part $\left(\theta>127^{\circ}\right)$ of the CAL. In addition, the trigger required an energy deposit in the FNC corresponding to $x_{L}>0.2$. The trigger efficiency of the FNC was close to $100 \%$ for the $x_{L}$ range under consideration in this paper $\left(x_{L}>0.64\right)$.

Photoproduction events were selected offline using cuts based on the reconstructed vertex position and calorimeter energy deposits. The events were required to have an energy in the LUMI electron calorimeter in the range $10<E^{\prime}<18 \mathrm{GeV}$ and an energy in the LUMI photon calorimeter smaller than $1 \mathrm{GeV}$ to eliminate bremsstrahlung overlap events. These cuts restricted the photon virtuality, $Q^{2}$, to values smaller than $\sim 0.02 \mathrm{GeV}^{2}$, with a median value of $\approx 10^{-3} \mathrm{GeV}^{2}$, and resulted in a mean value for $W$ of $220 \mathrm{GeV}$.

Events with a leading neutron were selected offline by requiring $x_{L}>0.64$ and by imposing the following cleaning cuts. Scattered protons, bent into the top towers 11 to 14 of the FNC (Fig. 1) by the HERA dipole magnets, were eliminated by requiring that the tower with the maximum energy deposit be in the range 6 to 9 . Photons were removed by eliminating showers with a small energy-weighted vertical width.

Neutrons that started showering in front of the FNC were removed by requiring that the scintillator veto counter farthest from the FNC had an energy deposit below that of a minimum-ionizing particle. To minimize false vetoes due to calorimeter albedo, only the farthest counter was used.

Finally, only events with $x_{L}<0.925$ were used in the analysis, since the effect of any error in the energy scale, and the influence of exchanges other than the pion, become significant at high $x_{L}$ (see Sections 5 and 6, respectively). After these selection cuts, the data sample consisted of 31756 events.

Not all neutrons start to shower in front of the FNT. To select events with useful position information, it was required that the energy deposited in each plane of the FNT be well above pedestal and that the strips with the two largest energy deposits be adjacent. These cuts reduced the sample to 17919 events.

\section{Neutron efficiencies and correction factors}

The efficiencies and correction factors for the leading neutron were calculated with a single-particle Monte Carlo (MC) simulation. The MC program included the geometry of the proton beam-line magnets which define the geometric acceptance, the details of the 
absorbing material as obtained from survey measurements, the proton beam divergence, and the measured energy (FNC) and position (FNT) resolutions for hadronic showers. The MC program accounts for the different amount of absorbing material in front of the FNC for the two cases when the ZEUS LPS was, or was not, in the data-taking position. Events were generated using the observed $x_{L}$ spectrum and an exponential distribution in transverse momentum, $e^{-b\left(x_{L}\right) p_{T}^{2}}$. The slope $b\left(x_{L}\right)$ was iterated until the MC distributions matched the observed uncorrected distributions. The corrected data distributions were obtained using bin-by-bin unfolding. The two samples of data, corresponding to the two cases when the LPS was, or was not, in the data-taking position, were corrected independently.

The simulation of the absorbing material was cross-checked by producing a material map from an analysis of low-energy $\left(x_{L}<0.27\right)$ neutron data, under the assumption that low $x_{L}$ neutrons are distributed uniformly over the geometric acceptance. This assumption is based on noting that both the maximum $p_{T}$ accepted by the FNC as well as the slope $b$ decrease with $x_{L}$ [3], and hence the geometric acceptance covers a small region of a slowly changing $p_{T}$ distribution. An analysis using this material map gives results consistent with those obtained using the material measurements from the survey.

The proton beam had a transverse momentum spread of approximately $0.04 \mathrm{GeV}$ horizontally and $0.1 \mathrm{GeV}$ vertically, as measured by the LPS. The variation of the proton beam orbit was considerably smaller than this spread. The spread broadens the observed position distribution of the neutrons in the FNC, but does not change the peak position.

\section{$5 \quad$ Results}

The events were binned in $x_{L}$ and $t$. The bins were chosen to be well within the acceptance in the $x_{L}-t$ plane and contained 12523 events. The corrected $x_{L}$ distributions of leading neutrons as a function of $t$ are shown in Fig. 2. The distributions are consistent with a power-law dependence in $\left(1-x_{L}\right)$ of the form $d N / d x_{L} \propto\left(1-x_{L}\right)^{a(t)}$. For each $t$ bin the power $a(t)$ was obtained by a least-squares fit of this function to the observed distribution. Only statistical errors were used in the fits because the systematic uncertainties are highly correlated. The resulting fits are superposed on the measured data points.

There are two main sources of systematic uncertainty: an uncertainty of $\pm 2 \%$ in the absolute energy scale of the FNC resulting from the calibration procedure $[3,12,13]$, and an uncertainty of $\pm 0.2 \mathrm{~cm}$ in each of the $X$ and $Y$ coordinates of the zero-degree point determined from the peak in the $X, Y$ distribution in the FNT [14]. The systematic uncertainties on $a(t)$ were obtained by varying the energy scale and beam-spot position within 
their uncertainties and then repeating the complete analysis. The dominant contribution comes from the energy-scale uncertainty.

The powers determined from the fits to the data in Fig. 2 are plotted as a function of $-t$ in Fig. 3. They are consistent with a linear function,

$$
\left.a(t)=0.88 \pm 0.09(\text { stat. })_{-0.39}^{+0.34} \text { (syst. }\right)-\left(2.81 \pm 0.42(\text { stat. })_{-0.62}^{+1.13}(\text { syst. }) \mathrm{GeV}^{-2}\right) t
$$

The correlation between the slope and the intercept is predominantly statistical; the coefficient of correlation is -0.9 .

\section{Discussion}

Previous experiments ([3] and references therein) have shown that leading neutron production in lepton-hadron and hadron-hadron experiments can be described by pion-exchange models. The consistency of this description can be tested by assuming that pion exchange is the dominant mechanism and deriving the pion Regge trajectory from the measured values of $a(t)$.

The pion "flux", the splitting function of a proton to a neutron and pion $\left(p \rightarrow n \pi^{+}\right)$, can be written [15] as

$$
f_{\pi / p}\left(x_{L}, t\right)=\frac{1}{4 \pi} \frac{g_{n \pi p}^{2}}{4 \pi} \frac{-t}{\left(m_{\pi}^{2}-t\right)^{2}}\left(1-x_{L}\right)^{1-2 \alpha_{\pi}(t)}\left(F\left(x_{L}, t\right)\right)^{2},
$$

where $g_{n \pi p}$ is the coupling at the $n \pi p$ vertex, $m_{\pi}$ is the mass of the pion, and $\alpha_{\pi}(t)=$ $\alpha_{\pi}^{\prime}\left(t-m_{\pi}^{2}\right)$ is the Regge trajectory of the pion [16]. The function $F\left(x_{L}, t\right)$ is a form-factor which accounts for the effect of hadronic structure on the $p \rightarrow \pi n$ amplitude and for final-state rescattering of the neutron. As discussed elsewhere [2], this prescription for the flux with $F\left(x_{L}, t\right)$ set to 1 describes most of the $p n \rightarrow X p$ data and also gives a good description of the ZEUS $x_{L}$ spectrum for photoproduced neutrons [2,3]. In this analysis it is assumed that $F\left(x_{L}, t\right)$ is a function of $t$ only.

The flux is thus of the form $f_{\pi / p}=A(t)\left(1-x_{L}\right)^{1-2 \alpha_{\pi}(t)}$. The cross section for neutron production is given by the product of the flux and the total $\gamma \pi$ cross-section $\sigma_{\gamma \pi}\left(s^{\prime}\right)=$ $\sigma_{\gamma \pi}\left(\left(1-x_{L}\right) W^{2}\right)$ as

$$
\frac{d^{2} \sigma}{d x_{L} d t}=A(t)\left(1-x_{L}\right)^{1-2 \alpha_{\pi}(t)} \sigma_{\gamma \pi}\left(\left(1-x_{L}\right) W^{2}\right)
$$

where $s^{\prime}=\left(1-x_{L}\right) W^{2}$ is the square of the $\gamma \pi$ center-of-mass energy. The total $\gamma \pi$ cross section is assumed to have a power law dependence on $s^{\prime}$, for large $s^{\prime}$, of the DonnachieLandshoff form [17]

$$
\sigma_{\gamma \pi}\left(s^{\prime}\right)=\mathcal{A}\left(s^{\prime}\right)^{\epsilon}+\mathcal{B}\left(s^{\prime}\right)^{-\eta},
$$


where $\epsilon \approx 0.1$ and $\eta \approx 0.5$. The two terms correspond to the exchange of the Pomeron $(\mathbb{P})$ and the Reggeon $(\mathbb{R})$, respectively. For large $s^{\prime}$ the Pomeron contribution dominates that of the Reggeon. In this case the $\left(1-x_{L}\right)$ distribution is proportional to $\left(1-x_{L}\right)^{a(t)}$, where

$$
a(t)=1+\epsilon-2 \alpha_{\pi}^{\prime} t .
$$

Here $m_{\pi}^{2}$ has been ignored, since its effect on the result is much smaller than the systematic error. Therefore, the measured linear function $a(t)$ discussed in Section 5 has an intercept of $\alpha_{\mathbb{I P}}(0)=(1+\epsilon)$, the value of the Pomeron trajectory at $t=0$, and a slope of $2 \alpha_{\pi}^{\prime}$, twice the slope of the pion trajectory.

It is assumed that the $\mathbb{P}$ term dominates in the $x_{L}$ region of this measurement. The fit [17] to the total $\gamma p$ cross section suggests that the highest contribution of the $\mathbb{R}$ term is about $12 \%$ at $x_{L}=0.925$. As $x_{L}$ decreases ( $s^{\prime}$ increases), the Reggeon contribution falls rapidly. No correction for the Reggeon contribution is applied in this analysis.

Previous measurements of the effective Regge trajectory in the $p \rightarrow n$ transition in hadronic interactions $[16,18,19]$ found it to be dominated by the pion, with the intercept in the range -0.1 to 0.3 and the slope in the range 1.1 to $1.3 \mathrm{GeV}^{-2}$.

The values extracted from the experimental results,

$$
1+\epsilon=\alpha_{\mathbb{P}}(0)=0.88 \pm 0.09(\text { stat. })_{-0.39}^{+0.34}(\text { syst. })
$$

and

$$
\alpha_{\pi}^{\prime}=1.40 \pm 0.21 \text { (stat.) }{ }_{-0.31}^{+0.56} \text { (syst.) } \mathrm{GeV}^{-2}
$$

are consistent with the expectation that $\alpha_{\mathbb{P}}(0)$ is about 1.1 and $\alpha_{\pi}^{\prime}$ is about $1 \mathrm{GeV}^{-2}$ in the range of $0.08<-t<0.425 \mathrm{GeV}^{2}$, and so support the hypothesis that pion exchange is the dominant process in this reaction. Note that the data rule out a significant role for $\rho$ exchange. If the $\rho$ trajectory, $\alpha_{\rho}=0.5+t[20]$, is substituted for the pion trajectory in Eq. (1), the value for $1+\epsilon$ extracted from the data is 1.88 rather than 0.88 , which is an inconsistent result for $\alpha_{\mathbb{P}}(0)$.

\section{Summary}

The dependence of the energy distribution of photoproduced leading neutrons on the momentum transfer at the proton-neutron vertex has been studied at an average photonproton center-of-mass energy of $220 \mathrm{GeV}$. The $\left(1-x_{L}\right)$ distributions in bins of $t$ are described by a power law, $d N / d x_{L} \propto\left(1-x_{L}\right)^{a(t)}$, with the powers $a(t)$ following a linear function of $t: a(t)=0.88 \pm 0.09$ (stat. ${ }_{-0.39}^{+0.34}$ (syst.) $-\left(2.81 \pm 0.42\right.$ (stat. ${ }_{-0.62}^{+1.13}$ (syst.) $\left.\mathrm{GeV}^{-2}\right) t$. 
The linear function can be interpreted in the framework of Regge theory. The measured values of the intercept and slope are in agreement with the expectations from pion exchange: i.e., the intercept is the value of the Pomeron trajectory at $t=0$, and the slope is twice the slope of the pion trajectory. The data thus confirm that the production of leading neutrons in photon-proton collisions is well described by the pion-exchange model.

\section{Acknowledgments}

We thank the DESY Directorate for their strong support and encouragement, and the HERA machine group for their diligent efforts. We are grateful for the support of the DESY computing and network services. The design, construction and installation of the ZEUS detector have been made possible owing to the ingenuity and effort of many people who are not listed as authors. This study was only made possible by the physics insight and work of G. Levman, to whom we are greatly indebted. 


\section{References}

[1] ZEUS Coll., M. Derrick et al., Phys. Lett. B 384, 388 (1995).

[2] ZEUS Coll., J. Breitweg et al., Nucl. Phys. B 596, 3 (2001).

[3] ZEUS Coll., S. Chekanov et al., Nucl. Phys. B 637, 3 (2002).

[4] ZEUS Coll., S. Chekanov et al., Phys. Lett. B 590, 143 (2004).

[5] H1 Coll., C. Adloff et al., Eur. Phys. J. C 6, 587 (1999).

[6] N. Harnew et al., Nucl. Inst. Meth. A 279, 290 (1989);

B. Foster et al., Nucl. Phys. Proc. Suppl. B 32, 181 (1993);

B. Foster et al., Nucl. Inst. Meth. A 338, 254 (1994).

[7] M. Derrick et al., Nucl. Inst. Meth. A 309, 77 (1991);

A. Andresen et al., Nucl. Inst. Meth. A 309, 101 (1991);

A. Caldwell et al., Nucl. Inst. Meth. A 321, 356 (1992);

A. Bernstein et al., Nucl. Inst. Meth. A 336, 23 (1993).

[8] J. Andruszków et al., Preprint DESY-92-066, DESY, 1992;

ZEUS Coll., M. Derrick et al., Z. Phys. C 63, 391 (1994);

J. Andruszków et al., Acta Phys. Pol. B 32, 2025 (2001).

[9] ZEUS Coll., M. Derrick et al., Z. Phys. C 73, 253 (1997).

[10] S. Bhadra et al., Nucl. Inst. Meth. A 354, 479 (1995).

[11] S. Bhadra et al., Nucl. Inst. Meth. A 394, 121 (1997).

[12] S. Bhadra et al., Proc. of the Seventh International Conference on calorimetry in High Energy Physics, Tuscon, Arizona, November 1997, E. Cheu et al. (ed.), p. 295. World Scientific, Singapore (1998).

[13] C.-P. Fagerstroem. Ph.D. Thesis, University of Toronto (1999) (unpublished).

[14] T. Koop. Ph.D. Thesis, University of Toronto (2004) (unpublished).

[15] M. Bishari, Phys. Lett. B 38, 510 (1972).

[16] H. Abramowicz et al., Nucl. Phys. B166, 62 (1980).

[17] A. Donnachie and P. V. Landshoff, Phys. Lett. B 296, 227 (1992).

[18] J. Hanlon et al., Phys. Rev. D20, 2135 (1979).

[19] W. Flauger and F. Mönnig, Nucl. Phys. B109, 347 (1976).

[20] R. J. Eden, Rep. Prog. Phys. 34, 995 (1971). 


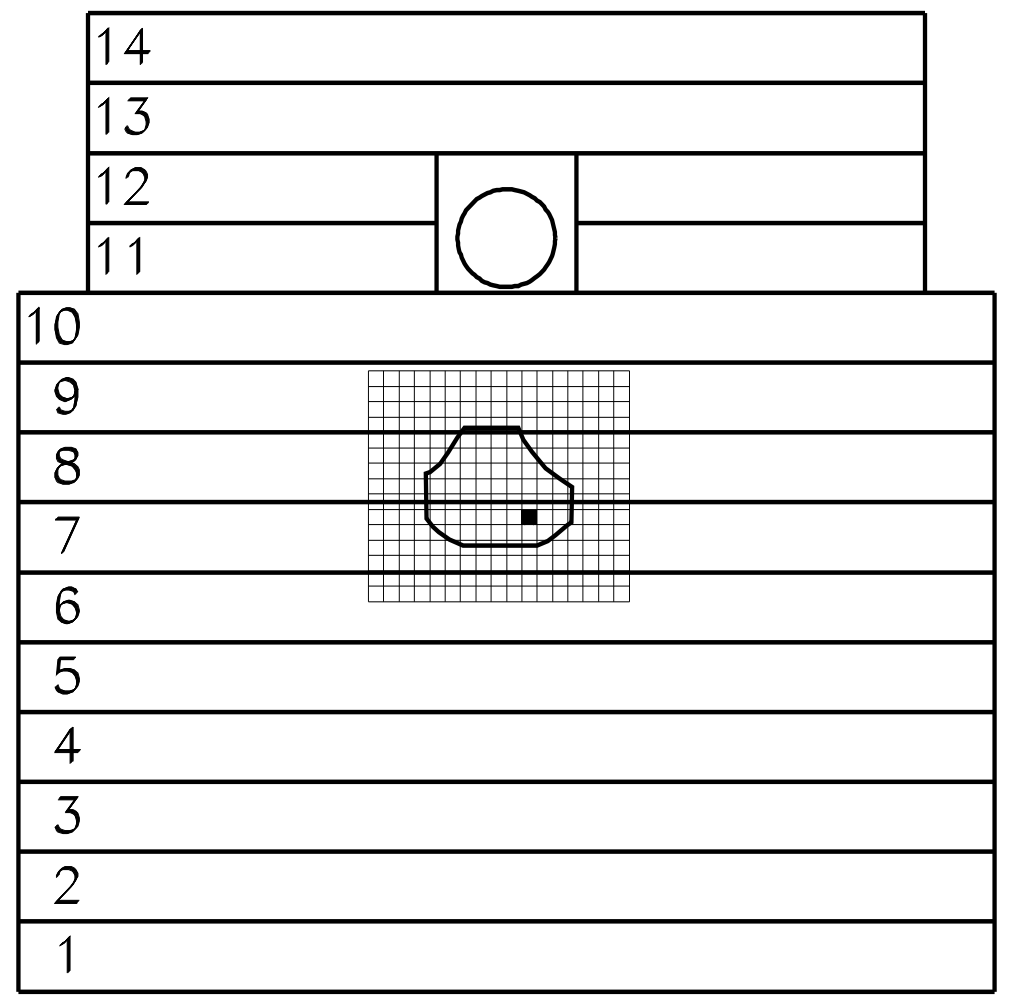

Figure 1: The FNC as viewed from the rear with the tower numbers indicated on the left-hand side. The scintillator strips of the FNT hodoscope are shown superposed on towers 6-9 of the FNC. The irregular contour shows the outline of the geometric acceptance allowed by the proton beam-line elements. The full square indicates the approximate position of the projection of the zero-degree line. The proton beampipe, shown as a circle, passes through a hole in the upper part of the FNC. 


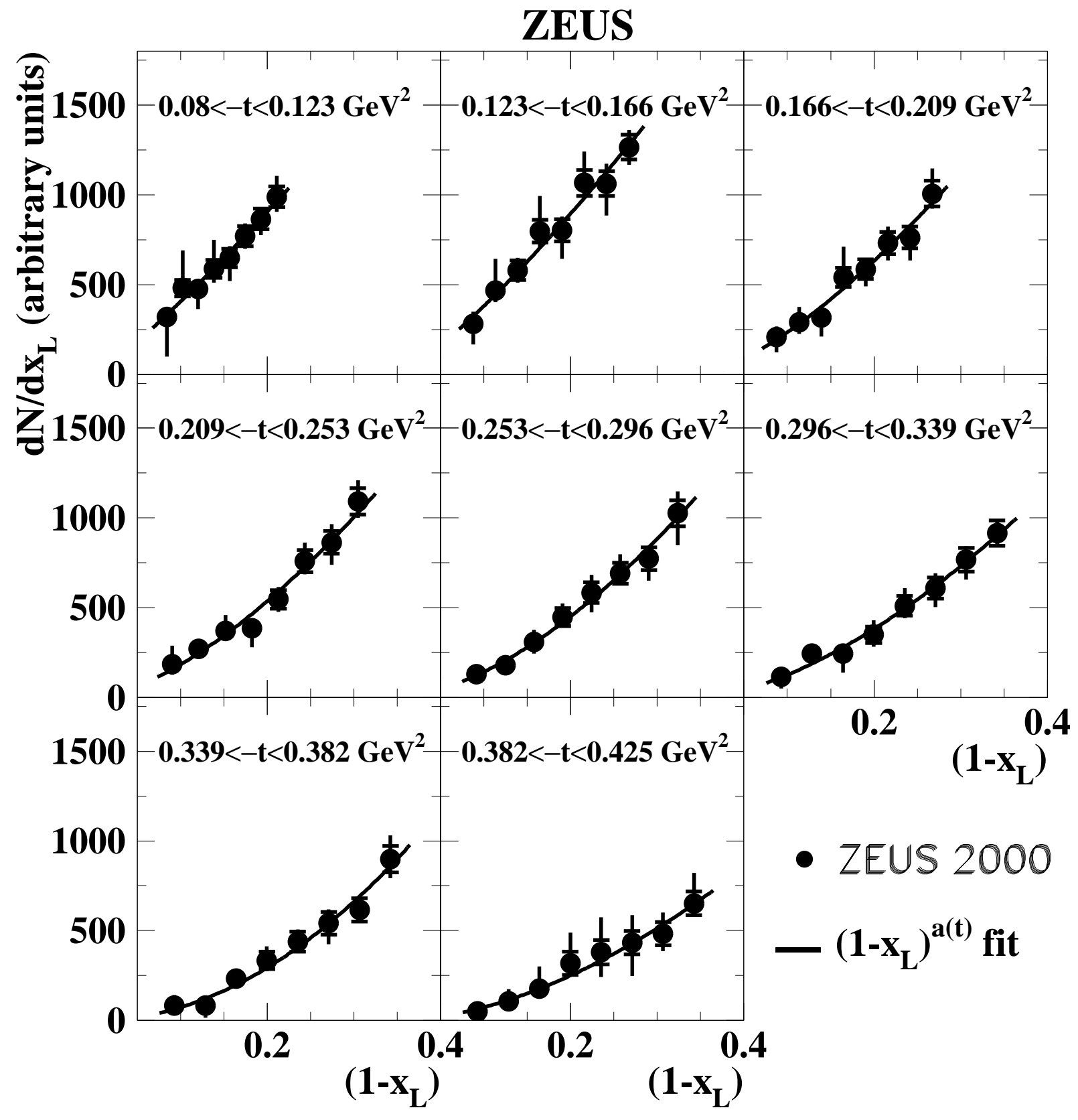

Figure 2: $\quad$ The $x_{L}$ distribution in bins of $t$. The curves show the results of fits to the data with $0.64<x_{L}<0.925$ to the form $\left(1-x_{L}\right)^{a(t)}$. The inner error bars indicate the statistical uncertainties and the full bars indicate the quadratic sum of the statistical and systematic uncertainties. 


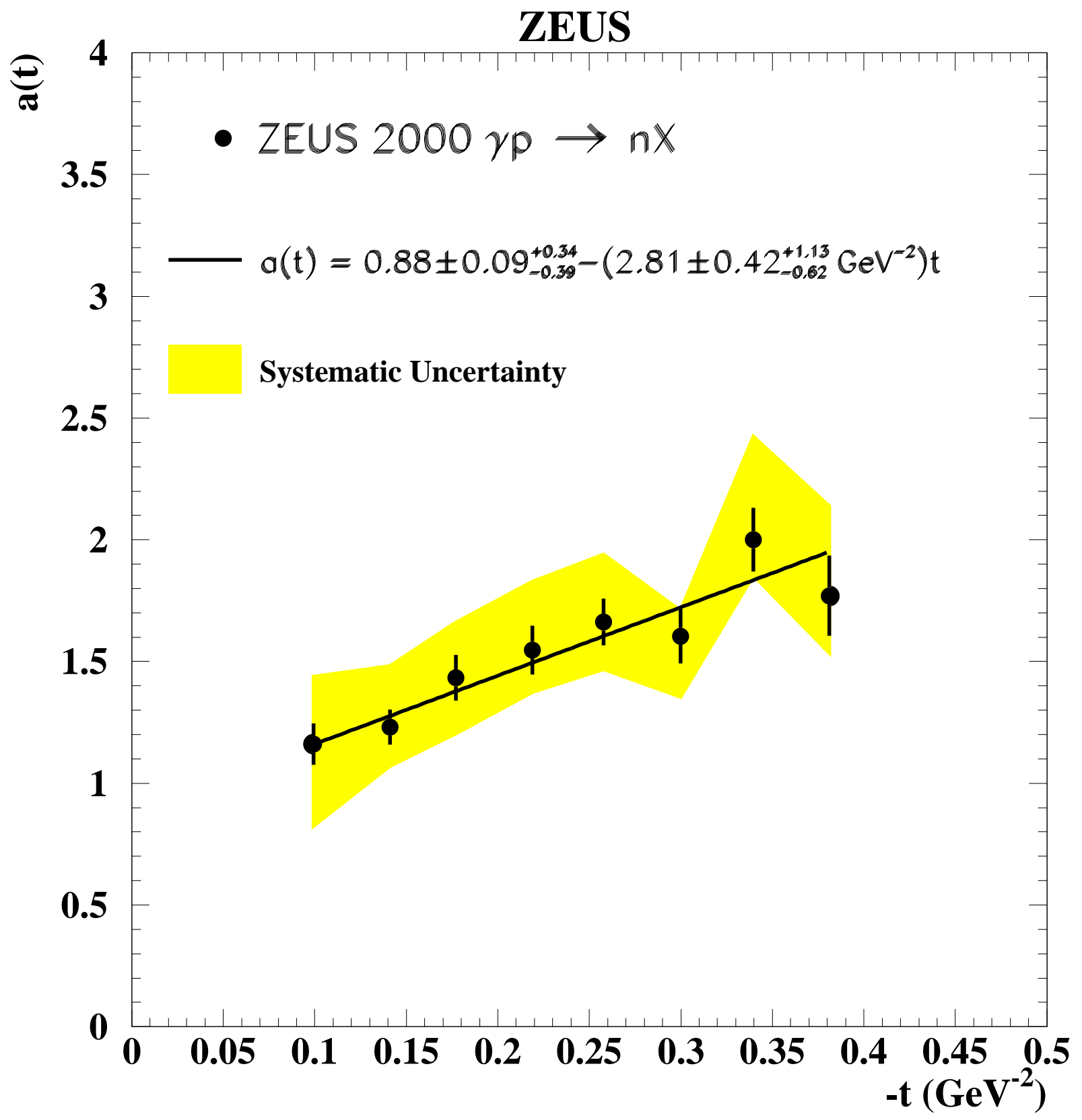

Figure 3: $\quad$ The powers, a $(t)$, with statistical errors, as a function of $-t$. The solid line is the result of the fit described in the text. The shaded band shows the systematic uncertainty. 